\title{
En route vers une nouvelle mobilité
}

Écrit par : Harri Pursiainen, Permanent Secretary, Ministry of Transport and Communications of Finland

Dernière mise à jour : 2 mars 2020

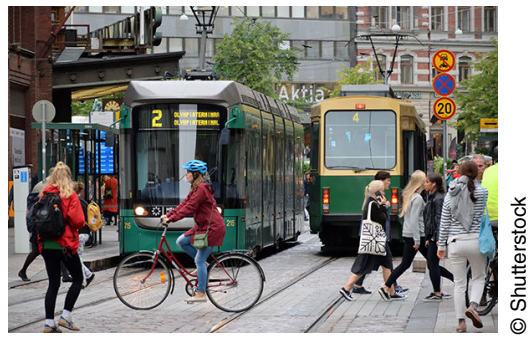

En route vers une nouvelle mobilité

Transformation numérique, changement climatique et urbanisation modifient nos vies et nous obligent à trouver de nouvelles manières de transporter les biens et les personnes. Nous devons réduire nos émissions de carbone et rendre la circulation plus sûre et plus fluide. Les outils numériques peuvent nous y aider. Après tout, des appareils de la taille d'une carte bancaire nous permettent de transférer des données quasi instantanément et d'accéder à l'internet partout dans le monde. Les technologies de téléphonie mobile nous offrent des services dont nous n'aurions pas même osé rêver il y a quelques années.

Aujourd'hui, j'aimerais que le secteur des transports connaisse la même évolution radicale, et je pense que le numérique nous permettra de le transformer pour créer un système de mobilité durable, efficace et centré sur l'usager.

\section{Des données aux services}

Les données sont la clé de voute de la transformation de notre système de transport et la nouvelle législation décisive adoptée par la Finlande en la matière ouvre la voie. La Finlande est le premier pays du monde à légiférer pour imbriquer tous les moyens de transport, du taxi au tramway urbain en passant par le train grande ligne et les vélos en libre service. Le transport des biens et des personnes d'un point $\mathrm{A}$ à un point $\mathrm{B}$ s'effectue ainsi de la manière la plus douce possible. 
Pour la loi finlandaise sur les services de transport, tout le système doit être perçu comme une entité unique.

Cette loi exige que tous les opérateurs de transport autorisent l'accès à leurs données essentielles (informations sur les itinéraires, les arrêts, les horaires, les tarifs, les disponibilités et l'accessibilité) dans un format lisible par machine, par l'ouverture de leurs interfaces. Avec le partage des données, les opérateurs de transport de biens et de personnes peuvent faire un usage plus efficace de leur flotte de véhicules.

En vertu de la loi, les opérateurs doivent aussi disposer de systèmes compatibles et s'accorder mutuellement l'accès aux interfaces de leurs systèmes de billetterie. Les pouvoirs publics les incitent à se conformer à ces dispositions en faisant de l'interopérabilité un critère des commandes publiques. Les opérateurs peuvent vendre des billets pour divers moyens de transports ; dans une gare, l'usager peut ainsi acheter un billet de train et un ticket de bus couvrant le reste du trajet. Se rendre d'un point $\mathrm{A}$ à un point $\mathrm{B}$ devient un jeu d'enfant. Avec cette solution appelée Mobility as a Service (« la mobilité comme service »), ou MaaS, la notion de déplacement traditionnel et de modes de transport indépendants et coupés les uns des autres est supplantée par la notion de service pensé pour le consommateur - un service en principe optimal. Ce principe est au cœur de la nouvelle loi sur les transports.

Entrée en vigueur en juillet 2018 pour l'essentiel, la loi sur les transports a, jusqu'à présent, largement répondu à nos attentes. Conformément aux règles concernant les données inscrites dans la loi, les fournisseurs de services de mobilité ont ouvert l'accès à un grand nombre d'interfaces afin de permettre l'échange de données essentielles. L'ouverture des interfaces de vente a également commencé. Les opérateurs ont signalé que de nouveaux services et de nouveaux systèmes avaient été rapidement mis en place : un début prometteur !

La MaaS facilite les déplacements des biens et des personnes mais entraînera aussi une baisse des émissions de carbone. Ce nouveau système transforme la mobilité en une chaîne de transport qui combine transports publics et services privés, offrant ainsi une solution axée sur l'utilisateur, rentable, séduisante et durable.

Pourquoi durable ? Parce qu'un système qui permet de se déplacer d'un point A à un point $B$ en combinant métro, vélo en libre-service et taxi collectif apparaît comme une option avantageuse par rapport à la voiture. De plus, il incite l'usager à choisir les moyens de transport les moins polluants en veillant à ce que ceux-ci soient aussi les plus rapides et les plus économiques.

Même dans les zones rurales, la MaaS peut constituer une solution viable. Plusieurs projets pilotes porteurs cherchent à associer différents moyens de transport en collaboration avec les opérateurs publics et privés. Ainsi, le projet Open Arctic MaaS, en Laponie, et le projet Alpio, expérimentent tous deux 
différentes façons d'organiser les services de mobilité dans les zones à faible densité de population.

\section{Saurons-nous adopter une approche globale?}

La législation n'est qu'une partie de la solution. Si elle peut ouvrir la voie au changement, elle ne peut pas le faire advenir seule. Il faut, pour cela, une coopération entre les opérateurs publics et privés, mais aussi entre les différents secteurs et niveaux d'administration.

Bien que la solution finlandaise décrite ici soit locale, les défis à relever sont de dimension mondiale, et les réponses proposées doivent l'être aussi. Je suis confiant: nous pouvons trouver des solutions communes et adopter des pratiques exemplaires. Le projet horizontal de l'OCDE sur le numérique a prouvé que c'était possible dans de nombreux domaines touchés par la révolution numérique.

Nous devons rassembler le courage de changer nos pratiques les plus fondamentales, même si cette évolution ne plaît pas à tout le monde. Dans l'histoire des transports, bien des éléments qui nous paraissent aujourd'hui naturels sont partis d'idées radicales et audacieuses.

@L'Observateur de l'OCDE, mars 2019

\section{References:}

- Voir : https://maas.global/ https://maas.global/

- Voir : https://maas.global/ https://maas.global/

- Cet article fait partie d'une série consacrée à la célébration du 50e anniversaire de l'adhésion de la Finlande à l'OCDE : www.oecdobserver.org/ finland50oecd www.oecdobserver.org/finland50oecd 